\title{
An Analytical Model for Prioritized Contention Access in ECMA-368 MAC Protocol
}

\author{
Nasim Arianpoo, Yuxia Lin, Vincent W.S. Wong \\ Department of Electrical and Computer Engineering \\ The University of British Columbia, Vancouver, BC, Canada \\ e-mail: \{nasima, yuxial, vincentw\}@ece.ubc.ca
}

\author{
Attahiru Sule Alfa \\ Department of Electrical and Computer Engineering \\ University of Manitoba, Winnipeg, MB, Canada \\ e-mail: alfa@ee.umanitoba.ca
}

\begin{abstract}
The European Computer Manufacturers Association (ECMA) International recently defined the ECMA-368 standard, which specifies the physical and media access control (MAC) layers for Ultra Wideband (UWB) based wireless personal area networks (WPANs). The MAC protocol in ECMA-368 has a superframe structure. Each superframe is divided into three different time periods. One of them is the prioritized contention access (PCA) period which supports contention-based access between different traffic classes. In this paper, we propose an analytical model to evaluate the performance of PCA in ECMA368 MAC protocol. We assume that packets follow the Markovian Arrival Process (MAP) and various service times can be modeled by different phase type distributions (PHs). We apply the Matrix Geometric Method (MGM) technique and model the system as a MAP/PH/1 queueing system. We derive the probability mass function for the number of the packets in the queue, and the cumulative distribution function for the packets' waiting time. The correctness of our proposed analytical model is validated via OPNET simulations.
\end{abstract}

\section{INTRODUCTION}

Ultra Wideband (UWB) is an emerging technology for high rate, short range wireless communications. Its unique features such as low power operation, robustness to multi-path fading, and accurate positioning capabilities makes UWB a good platform for wireless personal area networks (WPANs). One of the recent UWB standards standardized by the European Computer Manufacturers Association (ECMA) International is the ECMA-368 [1], which defines the physical (PHY) and media access control (MAC) layers for high rate WPANs. The PHY layer uses multi-band orthogonal frequency division multiplexing (MB-OFDM) UWB technique. The MAC protocol in ECMA-368 has a superframe structure (see Figure 1). Each superframe is divided into three different time periods. The beacon period is used for control purposes. The distributed reserved protocol (DRP) period allows devices to reserve bandwidth for data transmission. The PCA (prioritized contention access) period supports contention-based access between different traffic classes.

The channel access during PCA period is similar to the channel access in IEEE 802.11e. Each node has to contend to obtain the transmission opportunity (TXOP). The PCA period provides service differentiation for four access categories (ACs). The service differentiation is provided with different Short Inter-Frame Spacing (SIFS[AC]), different transmission opportunity (TXOP[AC]) limit, and different minimum and

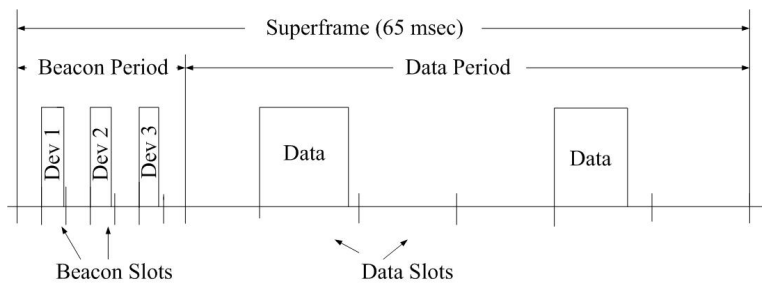

Fig. 1. Structure of a superframe in ECMA-368.

maximum contention window size $(\mathrm{CWmin}[\mathrm{AC}]$ and $\mathrm{CW}$ $\max [\mathrm{AC}])$ for each access category.

Various analytical models have been proposed to evaluate the performance of carrier sense multiple access with collision avoidance (CSMA/CA) based MAC protocols. Models that are based on the use of Markov chains can be found in [2], [3], [4], [5]. Another approach is to use the Matrix Geometric Method (MGM) [6]-[9]. The MGM method has been used to analyze the performance of MAC protocols in wireless networks [10] [11].

In this paper, we propose an analytical model to evaluate the performance of PCA in ECMA-368 MAC protocol. We assume that packets follow the Markovian Arrival Process (MAP) and various service times can be modeled by different phase type distributions (PHs). We apply the MGM technique and model the system as a MAP/PH/1 queueing system. The contributions of this paper are as follows:

- The model considers various input parameters including the packet arrival rates, packet collision due to channel contention, the number of devices in the system, and the buffer size for the packets waiting in the queue.

- We derive the number of packets in the system and the cumulative distribution function of the waiting time of the packets in the system.

- We validate the correctness of our analytical model via simulations. We develop the ECMA-368 MAC module by using the OPNET simulator. Analytical and simulation results are presented under different scenarios.

The rest of the paper is organized as follows. The mathematical background is provided in Section II. We describe our analytical model in Section III. Section IV presents the analytical and simulation results. Conclusions and future work are given in Section $\mathrm{V}$. 


\section{MAThematicAl BACKGROUND}

\section{A. Phase Type Distribution (PH)}

Consider a Markov chain of $n$ transient states and one absorbing state, the time to absorption is a random variable with a PH distribution. A PH distribution according to [12] can be represented with $(\alpha, T)$, where $\alpha$ is the initial probabilities of the transient states and $T$ is the matrix of probability transitions of the transient states. All the characteristics of a distribution can be defined with these two matrices. The probability transition matrix $P$ is as follows:

$$
P=\left[\begin{array}{cc}
T & T^{0} \\
0 & 1
\end{array}\right],
$$

where $T^{0}$ is the column vector of transition probabilities to the absorbing state. $T^{0}$ is defined as follows:

$$
T^{0}=\mathbf{1}-T \mathbf{1}
$$

where $\mathbf{1}$ is a column vector with all entries equal to one and it has the same dimension of $T$. Many distributions can be represented by $\mathrm{PH}$ distributions in discrete time domain. For example, the geometric distribution with parameter $q$ can be represented as follows:

$$
\begin{gathered}
T=[q], \\
\alpha=[1], \\
T^{0}=[1-q] .
\end{gathered}
$$

\section{B. Markovian Arrival Process}

A Markovian arrival process (MAP) with $n$ phases can be represented by four $n \times n$ matrices $D_{10}, D_{11}, D_{20}$, and $D_{21}$. The elements $\left(D_{10}\right)_{i j}$ and $\left(D_{11}\right)_{i j}$ represent transitions from state $i$ to $j$ with no arrival and one arrival, respectively, at the DRP queue. The elements $\left(D_{20}\right)_{i j}$ and $\left(D_{21}\right)_{i j}$ represent transitions from state $i$ to $j$ with no arrival and one arrival, respectively, at the PCA queue. The matrix $D=D_{10}+D_{11}+$ $D_{20}+D_{21}$ is stochastic. Let $\nu$ be defined as the solution of the following equations:

$$
\nu=\nu D
$$

and

$$
\nu \mathbf{1}=1 \text {. }
$$

Then, $\lambda_{1}$ and $\lambda_{2}$ which are the respective arrival rates to the DRP and PCA queues can be determined from:

$$
\lambda_{1}=\nu D_{11} \mathbf{1}
$$

and

$$
\lambda_{2}=\nu D_{21} \mathbf{1}
$$

\section{Matrix Geometric Method (MGM)}

Consider a MAP/PH/1 system. The probability transition matrix $P$ of the system is a Quasi Birth Death (QBD) process and is defined as follows:

$$
P=\left[\begin{array}{cccccc}
B & C & & & \\
E & A_{1} & A_{0} & & \\
& A_{2} & A_{1} & A_{0} & \\
& & \ddots & \ddots & \ddots &
\end{array}\right],
$$

where $A_{2}, A_{1}, A_{0}, B, C$, and $E$ are the matrix blocks which are determined according to the characteristics of the system such as the arrival process and service time distribution. One way to find the steady state probabilities of an infinite size Markov chain is to use the MGM. The concept of MGM is to use smaller matrix blocks such as $A_{0}, A_{1}$, and $A_{2}$ instead of manipulating the matrix $P$ for calculation. Although the matrices $A_{0}, A_{1}, A_{2}$ can sometimes be large, their dimensions are much smaller than the matrix $P$.

The matrix $A=A_{1}+A_{2}+A_{0}$ is a stochastic matrix. The Markov chain can be solved by MGM if:

$$
\pi A_{2} \mathbf{1}<\pi A_{0} \mathbf{1}
$$

where $\pi$ is the steady state probability of matrix $A$ and is calculated from:

$$
\pi=\pi A
$$

and

$$
\pi \mathbf{1}=1 \text {. }
$$

If the stability condition in (9) is satisfied, then the nonnegative matrix $R$ can be calculated as follows:

$$
R=A_{0}+R A_{1}+R^{2} A_{2} .
$$

The matrix $R$ has a spectral radius less than one [12] [13] [14]. The spectral radius of a matrix is defined as the largest eigenvalue of that matrix.

To make sure that the system is stable, there is another matrix $G$ which can be calculated as follows:

$$
G=A_{2}+A_{1} G+A_{2} G^{2} .
$$

The matrix $G$ is stochastic if the system is stable. From MGM [12] [13], we have:

$$
\mathbf{x}_{i+1}=\mathbf{x}_{i} R=\mathbf{x}_{1} R^{i}, \quad i=1,2, \ldots
$$

where $\mathbf{x}_{i}$ denotes the steady state probability vector of having $i$ packets in the system. To determine $\mathbf{x}_{1}$, we have to form another matrix $B[R]$, which is a function of the matrix $R$.

$$
B[R]=\left[\begin{array}{cc}
B & C \\
E & A_{1}+R A_{2}
\end{array}\right] .
$$

Let assume $\left[\begin{array}{ll}\mathbf{y}_{0} & \mathbf{y}_{1}\end{array}\right]$ be the eigenvector of $B[R]$ corresponding to the eigenvalue of 1 ; where $\mathbf{y}_{0}$ and $\mathbf{y}_{1}$ are the row vectors of the same dimension of $B$ and $E$, respectively. Since $B[R]$ 
is a stochastic matrix, it has an eigenvalue equal to one. $\mathbf{x}_{1}$ and $\mathbf{x}_{0}$ can be calculated as follows:

$$
\begin{gathered}
\mathbf{x}_{0}=y^{-1} \mathbf{y}_{0}, \\
\mathbf{x}_{1}=y^{-1} \mathbf{y}_{1}, \\
y=\mathbf{y}_{0} \mathbf{1}+\mathbf{y}_{1}(I-R)^{-1} \mathbf{1} .
\end{gathered}
$$

We can find other steady state probabilities from (14). The $\mathbf{x}_{i}$ vectors are the steady state probabilities of the matrix $P$.

\section{Kronecker Product}

Elaborating on MGM is not possible without considering the contribution of Kronecker product. In mathematics, the Kronecker product, $\otimes$, is an operator between two arbitrary matrices that results in a block matrix. For instance, assume matrices $A$ and $B$ are as follows:

$$
\begin{aligned}
& A=\left[\begin{array}{ll}
a_{11} & a_{12} \\
a_{21} & a_{22}
\end{array}\right], \\
& B=\left[\begin{array}{ll}
b_{11} & b_{12} \\
b_{21} & b_{22}
\end{array}\right] .
\end{aligned}
$$

The matrix $A \otimes B$, the Kronecker product of $A$ and $B$ is:

$$
A \otimes B=\left[\begin{array}{ll}
a_{11} B & a_{12} B \\
a_{21} B & a_{22} B
\end{array}\right] .
$$$$
\text { III. AnAlyticAl MODEL }
$$

We assume a MAP/PH/1 system, where the arrival process follows a MAP distribution, and the service time follows a PH distribution. The server has three different modes of service. The first mode is the beacon period during which no data packets are being transmitted. Therefore, the server can be considered as idle. The second mode is the DRP part, in which each node has allocated one time slot for data transmission. The distribution of the service time for this part can be modeled as a deterministic service. The third mode of the server is the PCA with the geometric distribution. The state space of the system can be defined as:

$$
\begin{aligned}
\Delta_{B} & =\left(i, j, k_{1}, k_{2}, r\right), \\
\Delta_{T} & =\left(i, j, k_{1}, k_{2}, l\right), \\
\Delta_{C} & =\left(i, j, k_{1}, k_{2}, u\right),
\end{aligned}
$$

where $i$ is the number of the packets in the PCA queue, $j$ is the number of packets in the DRP queue; $k_{1}$ and $k_{2}$ are the phase of arrival for the PCA and DRP queue, respectively, since there are two separate queues for DRP and PCA; $r$ is the number of time slots that the server is in idle mode $\left(0 \leq r \leq N_{B}\right)$; $l$ is the number of time slots that the server has spent in the DRP mode $\left(0 \leq l \leq N_{T}\right)$, and $u$ is the number of time slots that server has spent in the PCA mode $\left(0 \leq u \leq N_{C}\right)$. The vector $\Delta_{B}$ denotes the states that the server is either in the idle mode or the beacon period. The vector $\Delta_{T}$ denotes the states when the server visits the DRP queue, and the vector $\Delta_{C}$ denotes the states when the server visits the PCA queue.

The deterministic service in the discrete time can be represented as a $\mathrm{PH}$ distribution with the number of phases equal to the number of time slots that the server visits the queue of that specific mode. For instance, a deterministic service with four phases can be shown as follows:

$$
\begin{aligned}
& S=\left[\begin{array}{llll}
0 & 1 & 0 & 0 \\
0 & 0 & 1 & 0 \\
0 & 0 & 0 & 1 \\
0 & 0 & 0 & 0
\end{array}\right], \\
& \beta=\left[\begin{array}{llll}
1 & 0 & 0 & 0
\end{array}\right],
\end{aligned}
$$

where $S$ is the transition probability matrix and $\beta$ is the initial state probability [13]. We follow the conventional notation [15], [6], [7] and use $(T, \alpha)$ for the arrival distribution and $(S, \beta)$ for the service distribution.

We can obtain the steady state probability of the system from the transition matrix $P$ in [16], which is a QBD based on the changes to the DRP queue. Therefore, when we want to find the waiting time distribution of the PCA or the inner queue in the Markov chain, we have to find the probability transition matrix regarding to changes to PCA queue. The process to find the probability transition matrix for PCA queue is the same as DRP queue. If we assume the transition probability matrix of PCA is $Q$, then $Q$ has the following format as a QBD:

$$
Q=\left[\begin{array}{ccccc}
B^{P C A} & C^{P C A} & & & \\
E^{P C A} & A_{1}^{P C A} & A_{0}^{P C A} & & \\
& A_{2}^{P C A} & A_{1}^{P C A} & A_{0}^{P C A} & \\
& & \ddots & \ddots & \ddots \\
& & & &
\end{array}\right]
$$

where the matrix blocks $A_{2}^{P C A}, A_{1}^{P C A}$, and $A_{0}^{P C A}$ are probability matrices standing for decreasing the number of packets in the PCA queue by one, no change in the number of packets, and increasing the number of packets by one, respectively. The matrix $B^{P C A}$ is the initial probability matrix representing no change in the number of PCA packets. The matrix $C^{P C A}$ is the initial probability matrix for the states with an increase in the number of DRP packets, and the matrix $E^{P C A}$ is the initial probability matrix for a decrease by one in the number of PCA packets. The block matrices $A_{2}^{P C A}, A_{1}^{P C A}, A_{0}^{P C A}$ are as follows:

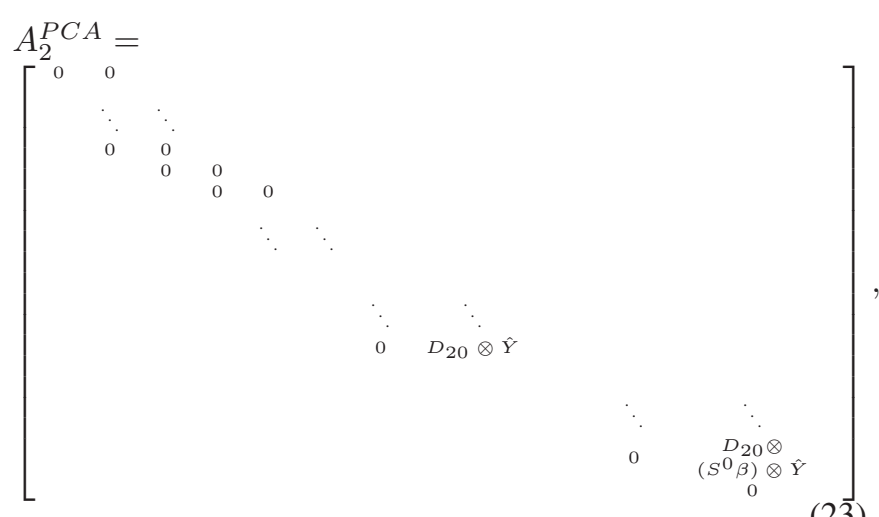




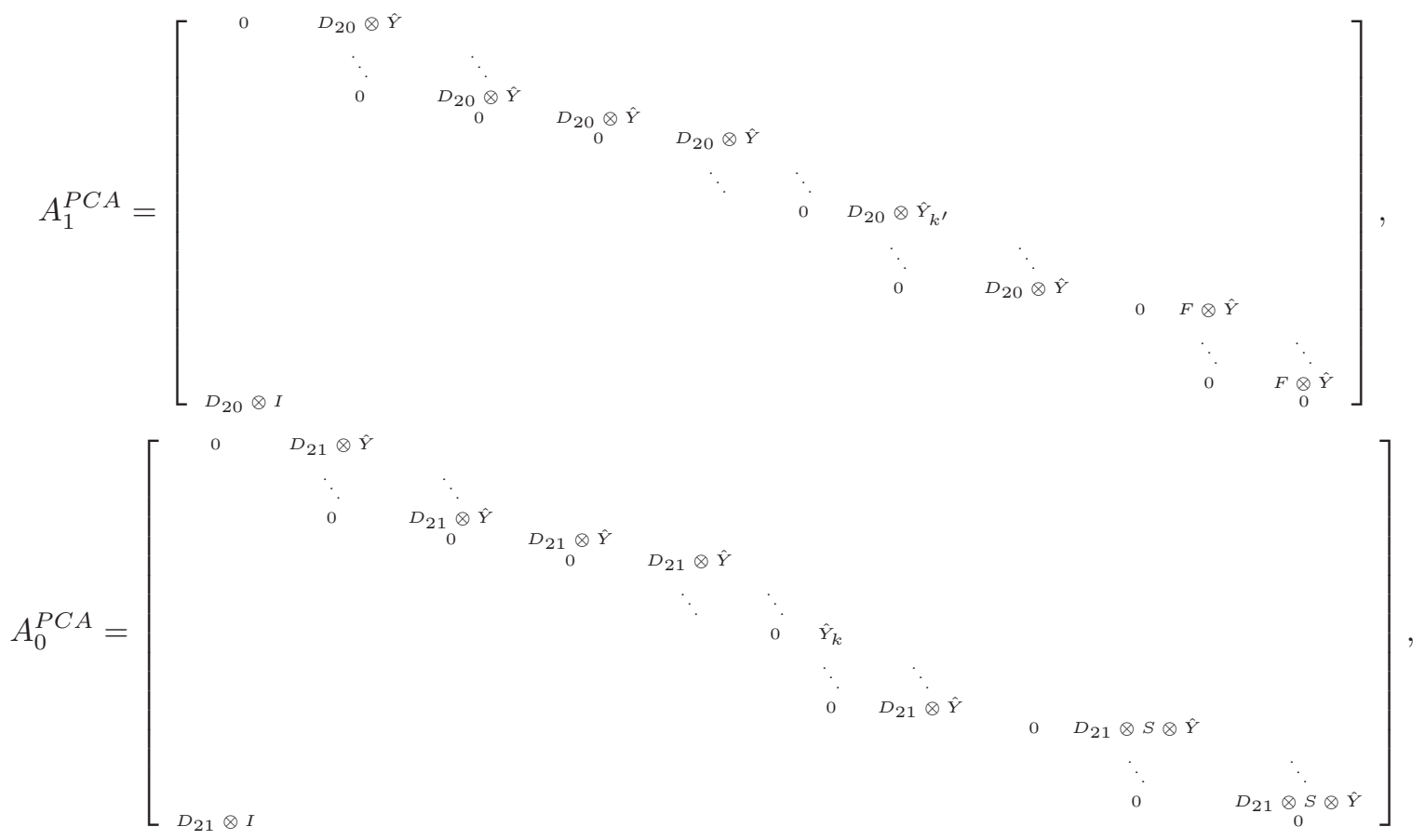

where $I$ is the identity matrix; $\hat{Y}_{k}$ is the probability of decreasing by one for the DRP queue and can be calculated as:

$$
\hat{Y}_{k}=\left[\begin{array}{cccc}
D_{20} & 0 & & \\
& \ddots & \ddots & \\
& & & 0 \\
& & & D_{21}
\end{array}\right],
$$

The matrix $\hat{Y}_{k^{\prime}}$ is defined as:

$$
\hat{Y}_{k^{\prime}}=\left[\begin{array}{cccc}
D_{21} & 0 & & \\
& \ddots & \ddots & \\
& & & 0 \\
& & & D_{21}
\end{array}\right],
$$

The matrix $\hat{Y}$ is defined as:

$$
\hat{Y}=\left[\begin{array}{cccc}
D_{20} & D_{21} & & \\
& \ddots & \ddots & \\
& & D_{20} & D_{21} \\
& & & D_{20}
\end{array}\right] .
$$

The matrix $F$ is defined as:

$$
F=\left[D_{20} \otimes S+D_{21} \otimes\left(S^{0} \beta\right)\right] .
$$

Now, we have the block matrices $A_{0}^{P C A}, A_{1}^{P C A}$ and $A_{2}^{P C A}$, matrix $R$ and the steady state probability vectors can be obtained from equations (12) and (14), respectively.

\section{A. Probability mass function (pmf) of the number of packets}

The probability mass function can be extracted from the steady state probability vectors that we obtained from (14). Let $q_{i}$ be the probability of having $i$ PCA packets in the system, then $q_{i}$ can be obtained as follows:

$$
q_{i}=\mathbf{x}_{i} \mathbf{1}
$$

where 1 is a column vector of one with the appropriate length. We can find the steady state probability vectors of the PCA from the model proposed in [16] as well.

\section{B. Waiting Time Distribution}

To determine the distribution of the waiting time of the system for each queue, we need to find the embedded Markov chain. The Markov chain that we implement for the system is based on a scenario from an observer to the system. To calculate the waiting time distribution, we need to find the state of the system from the point of view of a packet that arrives at the system. That is the reason why the embedded Markov chain needs to be modified. After the embedded Markov chain has been calculated, the steady state probability of the new chain can be determined.

After forming the probability transition matrix for the system based on the changes to the PCA queue, we can find the embedded Markov chain according to the number of packets in the line from an arriving packet point of view. According to [12], it is sufficient to find the steady state probability of the number of the packets in the PCA queue that a tagged packet sees as it arrives at the system.

Let $\hat{\mathbf{z}}_{j, u}^{B}$ denote the steady state probability vector of finding $j$ packets of PCA type in front of a tagged packet arrives at the system in time slot $u$ during the beacon period.

$$
\hat{\mathbf{z}}_{j, u}^{B}= \begin{cases}\hat{\mathbf{x}}_{j, N_{C}}^{C} D_{21}, & u=1, \\ \hat{\mathbf{x}}_{j, u-1}^{C} D_{21}, & u=2,3, \ldots, N_{B}\end{cases}
$$


TABLE I

PARAMETERS OF THE SIMULATOR

\begin{tabular}{|c|c|}
\hline Simulator Parameters & Value \\
\hline Data rate & $480 \mathrm{Mb} / \mathrm{s}$ \\
\hline Number of nodes & 5 \\
\hline DRP packet size & $15.25 \mathrm{kB}$ \\
\hline PCA packet size & $4.0 \mathrm{kB}$ \\
\hline Number of time slots in a superframe & 30 \\
\hline Number of time slots in the beacon period $\left(N_{B}\right)$ & 5 \\
\hline Number of time slots in the DRP period $\left(N_{T}\right)$ & 5 \\
\hline Number of time slots in the PCA period $\left(N_{C}\right)$ & 20 \\
\hline Duration of a time slot & $256 \mu \mathrm{s}$ \\
\hline Maximum number of DRP packets in the system & 20 \\
\hline Average packet arrival rate (PCA)-Poisson & 3125 Packets/sec \\
\hline Average packet arrival rate (DRP)-Poisson & 36 Packets/sec \\
\hline
\end{tabular}

where $N_{B}$ and $N_{C}$ are the number of beacon period time slots and PCA period time slots, respectively.

Let $\hat{\mathbf{z}}_{j, u}^{T}$ denote the steady state probability vector of finding $j$ packets of PCA type in front of a tagged packet arrives at the system in time slot $u$ during the DRP period.

$$
\hat{\mathbf{z}}_{j, u}^{T}= \begin{cases}\hat{\mathbf{x}}_{j, N_{B}}^{B} D_{21}, & u=1, \\ \hat{\mathbf{x}}_{j, u-1}^{B} D_{21}, & u=2,3, \ldots, N_{T}\end{cases}
$$

where $N_{T}$ is the number of time slots of the DRP period. Let $\hat{\mathbf{z}}_{j, u}^{C}$ denote the steady state probability vector of finding $j$ packets of PCA type in front of a tagged packet arrives at the system in time slot $u$ during the PCA period.

$$
\hat{\mathbf{z}}_{j, u}^{C}= \begin{cases}\hat{\mathbf{x}}_{j, N_{T}}^{T} D_{21}, & u=1, \\ \hat{\mathbf{x}}_{j, u-1}^{C}\left(D_{21} \otimes S\right) & \\ +\hat{\mathbf{x}}_{j+1, u-1}^{C}\left(D_{21} \otimes\left(S^{0} \beta\right)\right), & u=2,3, \ldots, N_{T}\end{cases}
$$

From the above steady state probabilities, we can calculate the waiting time distribution of the PCA packets. Let $\hat{\mathbf{z}}_{\mathbf{j}}=\left[\begin{array}{lll}\hat{\mathbf{z}}_{j}^{B} & \hat{\mathbf{z}}_{j}^{T} & \hat{\mathbf{z}}_{j}^{C}\end{array}\right]$ be the steady state probability vector of finding $j$ packets in front of a tagged packet arriving at the system, and each of $\hat{\mathbf{z}}_{j}^{B}, \hat{\mathbf{z}}_{j}^{T}, \hat{\mathbf{z}}_{j}^{C}$ is partitioned as $\hat{\mathbf{z}}^{\text {period }}=$

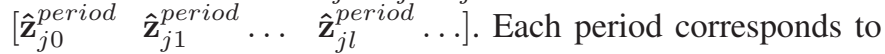
either beacon, PCA, or DRP. Then,

$$
\hat{\mathbf{z}}_{j+1}=\hat{\mathbf{z}}_{j} Q_{\text {embedded }}, \quad j=0,1,2, \ldots
$$

where $Q_{\text {embedded }}$ is the transition probability matrix of the embedded Markov chain that can be obtained from the transition probability matrix $Q$. The embedded Markov chain transition matrix can be obtained by replacing the $D_{20}$ with 0 , and $D_{21}$ with 1 in $Q$. The waiting time distribution can be obtained from:

$$
\hat{W}^{\tau}=\hat{\mathbf{z}}_{0}^{\tau} \mathbf{1}, \quad \tau=0,1, \ldots
$$

where $\hat{W}^{\tau}$ is the probability of waiting more than $\tau$ time slots in the PCA queue.

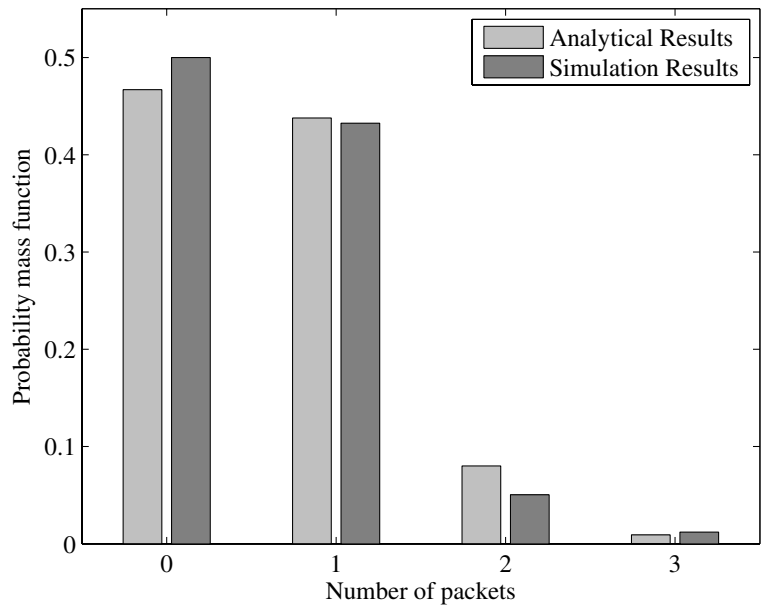

Fig. 2. Probability mass function of the number of PCA packets in a tagged node.

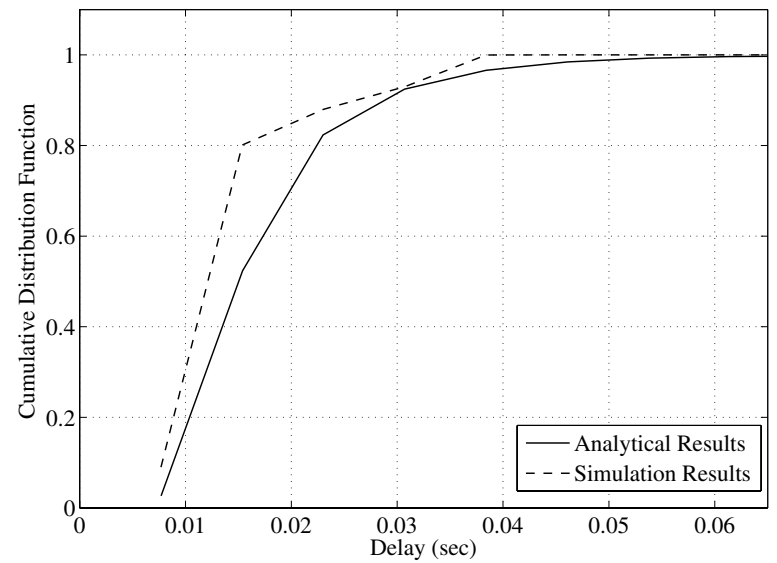

Fig. 3. Cumulative distribution function of the PCA packets waiting time.

\section{Results AND Discussion}

In this section, we present the analytical results and compare them with the simulation results to validate the correctness of our model. Table I shows the simulation parameters.

Figure 2 shows the pmf of the number of the PCA packets in a tagged node. Results from the analytical model and the simulation are consistent which show the correctness of the proposed analytical model. There is only a slight difference between the simulation and the analytical results. The reason behind is that in the simulation since the transmission of each packet may finish before the end of a specific time slot. Other nodes can sense the channel idle and wait for SIFS amount of time and start sending the next packet one time slot sooner than what happen in the analytical model. Since in the analytical model, the assumption is that the service will last for the whole time slot and the time slot is not going to be free before the end of the superframe. Therefore, for the cumulative distribution function of the waiting time of a packet in PCA queue (see Figure 3), there is a small gap between the analytical and simulation results. 


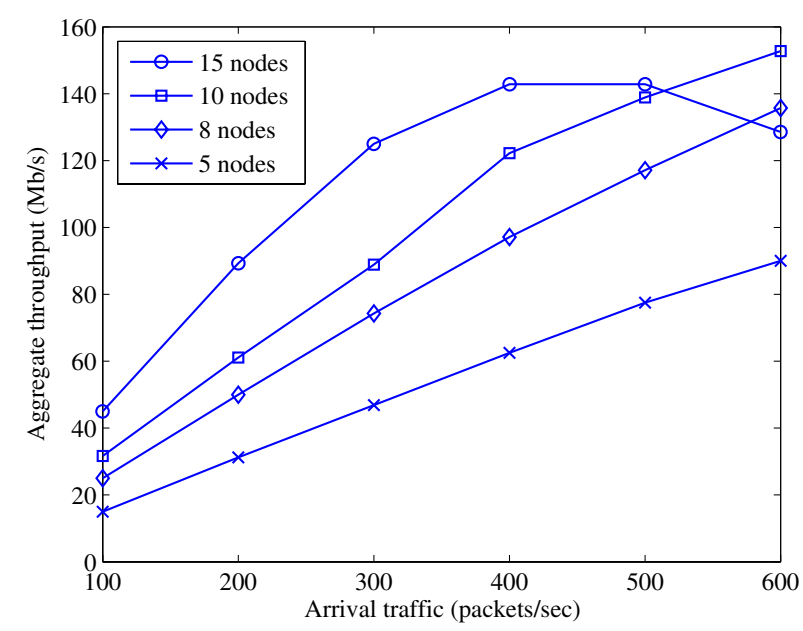

Fig. 4. Aggregate throughput of PCA traffic versus arrival traffic for different number of nodes in the network.

\section{A. Effect on Varying the Arrival Traffic}

In this section, we study the effect of changes in the arrival traffic of the network. Figure 4 shows the aggregate throughput versus the arrival traffic. As the number of the PCA packets which arrive at each node increases, the throughput increases. The throughput improvement continues until the network reaches its saturation condition. When the network is saturated, an increase of the traffic not only cannot increase the throughput but may also cause in a decrement in throughput to some extend. Figure 4 shows an increase in the number of nodes in the network can increase the aggregate throughput. The reason behind this increment is that when the number of the nodes increases, there is an increase in the arrival traffic of the network.

As the arrival traffic increases, the average amount of time that each packet should wait increases as well. Figure 5 shows the changes to the medium access delay due to the changes in the arrival traffic. In case of 15 nodes in the network, due to the increasing amount of collisions, the waiting time increases significantly. If the number of nodes within the network increases, the medium access delay will increase. The reason behind this increment is that there are higher number of nodes competing to obtain the channel. Therefore, on average each node should wait a longer time to obtain a TXOP.

\section{CONCLUSIONS}

In this paper, we proposed an analytical model for PCA in ECMA-368 MAC protocol. Based on the the MAP/PH/1 queueing model and MGM technique, we obtained the probability mass function of the number of packets in PCA queue and also the cumulative distribution function of the waiting time distribution of the packets in the PCA queue. We demonstrated that the results from the analytical model and the results from the OPNET simulator agreed with each other. For future work, we plan to extend the model by including different access categories in PCA and apply the MGM technique to analyze the IEEE $802.15 .3 a$ protocol.

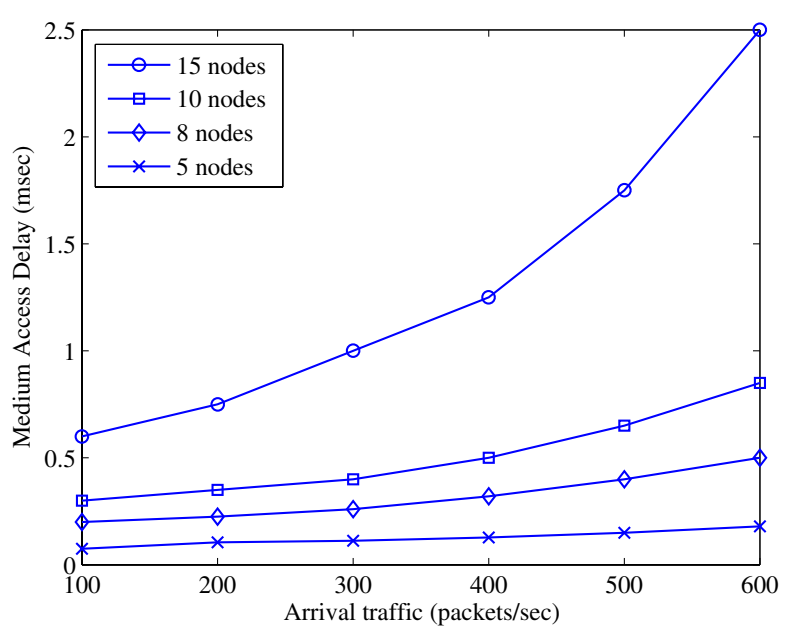

Fig. 5. Medium access delay of PCA traffic versus arrival traffic for different number of nodes in the network.

\section{ACKNOWLEDGMENT}

This work was supported by Bell Canada and the Natural Sciences and Engineering Research Council of Canada.

\section{REFERENCES}

[1] "ECMA-368: High rate ultra wideband PHY and MAC standard," Dec. 2005.

[2] G. Bianchi, "Performance analysis of the IEEE 802.11 distributed coordination function," IEEE Journal on Selected Areas in Communications, vol. 18, pp. 535-547, Mar. 2000.

[3] Z. Kong, D. Tsang, B. Bensaou, and G. Deyun, "Performance analysis of IEEE 802.11e contention-based channel access," IEEE Journal on Selected Areas in Communications, vol. 22, pp. 2095 - 2106, Dec. 2004.

[4] J. Zhao, Z. Guo, Q. Zhang, and W. Zhu, "Performance study of MAC for service differentiation in IEEE 802.11," in IEEE Globecom'02, Taipei, Taiwan, Nov. 2002

[5] Z. Tao and S. Panwar, "An analytical model for the IEEE 802.11e enhanced distributed coordination function," in Proc. of IEEE ICC'04, Paris, France, June 2004.

[6] A. Alfa, B. Liu, and Q. He, "Discrete-time analysis of MAP/PH/1 multiclass general preemptive priority queue," Naval Research Logistics, vol. 50, pp. 662-683, Dec. 2003.

[7] A. Alfa, "A discrete MAP/PH/1 queue with vacations and exhaustive time-limited service," Operations Research Letters, vol. 18, pp. 31-40, Aug. 1995.

[8] A. S. Alfa, "Vacation models in discrete time," Queueing Systems, vol. 44, pp. 5-30, Aug. 2003.

[9] — "Matrix-geometric solution of discrete time MAP/PH/1 priority queue," Naval Research Logistics, vol. 45, no. 1, pp. 23-50, Dec. 1998.

[10] A. Fallahi, E. Hossain, and A. S. Alfa, "QoS and energy trade off in distributed energy-limited mesh/relay networks: A queuing analysis," IEEE Trans. Parallel Distrib. Syst., vol. 17, pp. 576-592, June 2006.

[11] N. Arianpoo, Y. Lin, V. W. S. Wong, and A. S. Alfa, "Analysis of distributed reservation protocol for UWB-based WPANs with ECMA368 MAC," in Proc. of IEEE WCNC, Las Vegas, NV, March/April 2008.

[12] M. F. Neuts, Matrix Geometric Solution in Stochastic Models. The John Hopkins University press, 1981.

[13] A. Alfa, "Discrete time queues and matrix-analytic methods," TOP, vol. 10, no. 2, pp. 147-185, Dec. 2002.

[14] G. Latouche and V. Ramaswami, Introduction to Matrix Analytic Methods in Stochastic Modeling. American Statistical Association and Society for Industrial and Applied Mathematics, Jan. 1999.

[15] N. Akar, N. Oguz, and K. Sohraby, "A novel computational method for solving finite QBD processes," Communication in Statistic, Statistic Model, Mar. 2000.

[16] N. Arianpoo, "Performance evaluation of ECMA-368 medium access control protocol for UWB ad-hoc networks," Master's thesis, University of British Columbia, Vancouver, BC, Canada, Sept. 2007. 